\title{
Correction to: Discrepancies in Genetic Testing Procedures of BRCA1/2 Mutations: A National Survey Across China
}

\author{
Huanwen $\mathrm{Wu}^{1} \cdot$ Binghe $\mathrm{Xu}^{2} \cdot$ Qinglei Gao ${ }^{3} \cdot{\text { Xiaoyan } \mathrm{Zhou}^{4,5} \cdot \text { Jianyong Shao }}^{6,7} \cdot$ Zhiyong Liang $^{1} \cdot$ Ding Ma $^{3}$
}

Accepted: 7 January 2021 / Published online: 21 January 2021

(c) Springer Nature Switzerland AG 2021

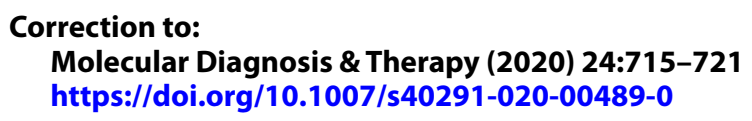

In the original publication of the article, text in Table 1 has a few errors as mentioned below.

Page 717, Table 1, 'Assessment criteria for quality scores of gBRCA/tBRCA tests of participating laboratories': The cell entry in column 2 , which previously read:

"Wrongly reported iItems" it should read as "Wrongly reported items"

Page 717, Table 1, 'Assessment criteria for quality scores of gBRCA/tBRCA tests of participating laboratories': The cell entry in column 1 , which previously read:

"pProtein" should read: "Protein"

Page 717, Table 1, 'Assessment criteria for quality scores of gBRCA/tBRCA tests of participating laboratories': The first cell entry in column 2 , which previously read:

"Class 3/class 2 misclassification" should read: "Class 3/ class 1 misclassification"

The corrected Table 1 is as given below:

The original article can be found online at https://doi.org/10.1007/ s40291-020-00489-0.

Zhiyong Liang

zhiyong829@gmail.com

$\triangle$ Ding Ma

dma@tjh.tjmu.edu.cn

1 Department of Pathology, Molecular Pathology Research Center, Peking Union Medical College Hospital, Chinese Academy of Medical Science and Peking Union Medical College, Beijing, China

2 National Cancer Center/Cancer Hospital, Chinese Academy of Medical Sciences and Peking Union Medical College, Beijing, China
3 Tongji Hospital, Tongji Medical College, Huazhong University of Science and Technology, Wuhan, China

4 Department of Pathology, Fudan University Shanghai Cancer Center, Shanghai, China

5 Department of Oncology, Shanghai Medical College; Institute of Pathology, Fudan University, Shanghai, China

6 Department of Molecular Diagnostics, Sun Yat-Sen University Cancer Center, Guangzhou, China

7 Sun Yat-Sen University Cancer Center, State Key Laboratory of Oncology in South China, Collaborative Innovation Center for Cancer Medicine, Guangzhou, China 
Table 1 Assessment criteria for quality scores of $\mathrm{g} B R C A / t B R C A$ test of participating laboratories

\begin{tabular}{lll}
\hline Assessment category & Wrongly reported items & Deduct marks \\
\hline Genotyping & Wrong gene & 2 \\
Nucleotide change & Wrong nucleotide change & 2 \\
& Wrong writing form & 0.5 \\
Protein change & Wrong protein change & 2 \\
& Wrong writing form & 0.5 \\
Homozygous or heterozygous (for germline testing) & Wrong results & 1 \\
Variant interpretation & Not shown & 1 \\
& Misclassification (false positive or false negative) & 2 \\
Others & Class 3/class 1 misclassification & 0.25 \\
& Class 3/class 2 misclassification & 0.5 \\
& VUS not detected or misreported & 0.5
\end{tabular}

